\section{(1) \\ CrossMark}

\title{
Prognostic value of right ventricular ejection fraction in pulmonary arterial hypertension
}

\author{
Pierre-Yves Courand ${ }^{1}$, Géraldine Pina Jomir ${ }^{2}$, Chahéra Khouatra ${ }^{3}$, \\ Christian Scheiber ${ }^{2}$, Ségolène Turquier ${ }^{4}$, Jean-Charles Glérant ${ }^{4}$, \\ Bénédicte Mastroianni ${ }^{3}$, Béatrice Gentil ${ }^{3}$, Anne-Sophie Blanchet-Legens ${ }^{3}$, \\ Alfred Dib ${ }^{3,5}$, Geneviève Derumeaux ${ }^{6}$, Marc Humbert ${ }^{7,8,9}$, \\ Jean-François Mornex ${ }^{3,5}$, Jean-François Cordier ${ }^{3,5}$ and Vincent Cottin ${ }^{3,5}$
}

Affiliations: ${ }^{1}$ Dept of Cardiology, Hospices Civils de Lyon, Croix-Rousse Hospital, Lyon, France. ${ }^{2}$ Dept of Nuclear Medicine, Hospices Civils de Lyon, Louis Pradel Hospital, Lyon, France. ${ }^{3}$ Dept of Respiratory Diseases, Hospices Civils de Lyon, Louis Pradel Hospital, Service de pneumologie - National Reference Centre for Rare Pulmonary Diseases, Regional Competence Center for Severe Pulmonary Arterial Hypertension, Lyon, France. ${ }^{4}$ Dept of Respiratory Physiology, Hospices Civils de Lyon, Louis Pradel Hospital, Lyon, France. ${ }^{5}$ Claude Bernard University Lyon 1, INRA, UMR754 INRA-Vetagrosup EPHE IFR 128, Lyon, France. ${ }^{6}$ Dept of Echocardiography, Hospices Civils de Lyon, Louis Pradel Hospital, Lyon, France. ${ }^{7}$ Univ. Paris-Sud, Le Kremlin-Bicêtre, France. ${ }^{8}$ AP-HP, Service de Pneumologie, DHU Thorax Innovation, Hôpital Bicêtre, Le Kremlin-Bicêtre, France. ${ }^{9}$ INSERM U999, LabEx LERMIT, Centre Chirurgical Marie Lannelongue, Le Plessis Robinson, France.

Correspondence: Vincent Cottin, Hospices Civils de Lyon, Hôpital Louis Pradel, Service de Pneumologie Centre de référence national des maladies pulmonaires rares, Lyon, France. E-mail: vincent.cottindachu-lyon.fr

ABSTRACT Right ventricle ejection fraction (RVEF) evaluated with magnetic resonance imaging is a strong determinant of patient outcomes in pulmonary arterial hypertension. We evaluated the prognostic value of RVEF assessed with conventional planar equilibrium radionuclide angiography at baseline and change 3-6 months after initiating pulmonary arterial hypertension-specific therapy.

In a prospective cohort of newly diagnosed patients with idiopathic, heritable or anorexigen-associated pulmonary arterial hypertension, RVEF was measured at baseline $(n=100)$ and 3-6 months after initiation of therapy $(\mathrm{n}=78)$. After a median follow-up of 4.1 years, 41 deaths occurred, including 35 from cardiovascular causes. Patients with a (median) baseline RVEF $>25 \%$ had better survival than those with a RVEF $<25 \%$ using Kaplan-Meier analysis $(p=0.010)$. RVEF at baseline was an independent predictor of all-cause and cardiovascular mortality in adjusted Cox regression model $(\mathrm{p}=0.002$ and $\mathrm{p}=0.007$, respectively; HR 0.93 for both). Patients with stable or increased RVEF at 3-6 months had a trend for improved all-cause survival (HR 2.43, $\mathrm{p}=0.086)$ and had less cardiovascular mortality $(\mathrm{HR} 3.25, \mathrm{p}=0.034)$ than those in whom RVEF decreased despite therapy.

RVEF assessed with conventional planar equilibrium radionuclide angiography at baseline and change in RVEF 3-6 months after therapy initiation independently predict outcomes in patients with pulmonary arterial hypertension.

@ERSpublications

RVEF assessed with CPERA at baseline, and its changes on therapy, independently predict outcome in patients with PAH http://ow.ly/DsCS4

For editorial comments see Eur Respir J 2015; 45: 33-35 [DOI: 10.1183/09031936.00209414].

This article has supplementary material available from erj.ersjournals.com

Received: Aug 282014 | Accepted: Oct 172014

Support statement: This study was funded by Fondation du souffle - Comité National contre les Maladies Respiratoires; Programme Hospitalier de Recherche Clinique; Hospices Civils de Lyon.

Conflict of interest: Disclosures can be found alongside the online version of this article at erj.ersjournals.com

Copyright CERS 2015 


\section{Introduction}

Pulmonary arterial hypertension (PAH) is characterised by increased pulmonary vascular resistance (PVR) and remodelling of the distal pulmonary arteries [1]. Medical therapies and advances in disease management have led to improved survival; however, $\mathrm{PAH}$ remains a progressive and fatal condition [2]. Biomarkers to assess prognosis and treatment response are eagerly needed. At baseline, the main predictors of survival have been found to be female, New York Heart Association (NYHA) functional class I and II, greater 6-min walking distance (6MWD), and two parameters associated with preserved right ventricle (RV) function, i.e. lower right atrial pressure and higher cardiac output [2]. As right heart failure is the main cause of death in patients with PAH [3], evaluation of RV function is deemed critical before the onset of overt clinical signs of RV failure, which heralds a poor prognosis despite intensive treatment [4]. Adaptation of the RV to the increase in right load (evaluated by PVR) may be a determinant for long-term outcome. However, discrepant trends can be observed between RV function and PVR, despite PAH-specific therapy [5], thereby suggesting that tools which specifically evaluate RV function may be useful [6].

Echocardiography and cardiac magnetic resonance imaging (MRI) allow noninvasive evaluation of RV function and structure, with several indices associated to prognosis [3]. Cardiac MRI can be used to assess RV ejection fraction (RVEF) [7]. A decreased stroke volume, increased RV end-diastolic volume and decreased RVEF at MRI predict a poor outcome in patients with $\mathrm{PAH}[5,8,9]$. However, cardiac MRI is not widely available in all centres, and its use may be limited by intensive and time-consuming data acquisition and analysis, with significant technical support and expertise needed. Echocardiography does not reliably evaluate RVEF, although other prognostic parameters have been validated. Nuclear medicine tomographic imaging techniques can also measure RVEF and can potentially be repeated at follow-up. However, neither conventional planar equilibrium radionuclide angiography (ERNA) nor single photon emission computed tomography (SPECT) have been evaluated in the setting of PAH. Measurements of RVEF using SPECT significantly correlate with RV function, evaluated with cardiac MRI [10]. Here, we hypothesised that a low baseline RVEF or a decrease in RVEF, despite initiation of PAH-specific therapy, may be associated with a poor outcome. Using single-centre data from a prospective PAH registry, we evaluated the prognostic value of RVEF measured using the widely available conventional planar ERNA method, both at baseline and after initiation of PAH-specific therapy.

\section{Methods}

Study design

All consecutive patients with PAH aged $\geqslant 18$ years were prospectively included in the French pulmonary hypertension (PH) registry [11]. Data from a single centre (Louis Pradel Hospital, Lyon, France) collected between June 1995 and September 2011 were extracted from the registry and patients satisfying the inclusion criteria were included in the present study. Only incident cases were included to avoid selection bias [12].

RVEF was measured, with conventional planar ERNA, as part of the routine evaluation in our centre (Louis Pradel Hospital) at baseline and during follow-up. This study was compliant with requirements of the Commission Nationale de l'Informatique et des Libertés. All patients provided informed consent to participate in the prospective registry and associated observational research protocols. This study was approved by the Institutional Review Board of the Société de Pneumologie de Langue Française (CEPRO 2012-001).

\section{Inclusion criteria}

The following inclusion criteria were applied. 1) Precapillary $\mathrm{PH}$, defined by mean pulmonary arterial pressure (PAP) $\geqslant 25 \mathrm{mmHg}$ and pulmonary artery wedge pressure $\leqslant 15 \mathrm{mmHg}$ at right heart catheterisation (RHC) [1]. 2) Group with idiopathic, familial, or anorexigen-associated PAH. 3) RVEF performed at baseline before initiation of PAH-specific therapy. 4) Available follow-up for at least 3 months (or death within 3 months). Patients with PH due to lung disease, including chronic obstructive pulmonary disease, idiopathic pulmonary fibrosis, and combined pulmonary fibrosis and emphysema, were excluded based on pulmonary function tests, arterial blood gas and chest high-resolution computed tomography (HRCT). Patients with associated conditions (e.g. congenital heart disease, HIV infection, portal hypertension and connective tissue disease) were excluded. Chronic thromboembolic $\mathrm{PH}$ was ruled out by ventilation perfusion scanning and HRCT of the chest. The date of diagnosis of PAH was that of the RHC.

\section{Investigations and management}

All data at baseline were obtained within 1 week of RHC and before initiation of PAH-specific therapy. Investigations were those routinely performed for patients with $\mathrm{PAH}$ in clinical practice according to current international and national guidelines [13, 14]. RHC was performed as described elsewhere [15]. Cardiac output was measured with the standard thermodilution technique. A vasodilator test with inhaled nitric oxide (10 ppm for 5-10 min) was carried out, and positive acute responses were defined as a decrease in mean PAP of $>10 \mathrm{mmHg}$ compared with baseline mean PAP, with mean PAP $<40 \mathrm{mmHg}$, and 
normal or increased cardiac output [15]. An unencouraged 6-min walk test was performed according to recommendations [16]. Treatment was left at the discretion of the physician, as per international and national guidelines and availability in France at the time of therapy initiation [13, 14]. Diuretics were added when clinical signs of RV failure were present. Patients with a positive acute response to inhaled nitric oxide were treated with high-dose calcium antagonists. All patients received oral anticoagulation unless contraindicated.

\section{Planar equilibrium radionuclide angiography}

An activity of $925 \mathrm{MBq}(25 \mathrm{mCi})$ of Tc99 m-pertechnetate was injected following an injection of $5 \mathrm{mg}$ stannous pyrophosphate for in vivo red blood cell labelling. Conventional planar ERNA [17] was performed in the optimal left anterior oblique projection, for best left and right ventricle septal separation. ECG-synchronised gated pictures (16 frames per cardiac cycle, $64 \times 64$ matrices) were acquired using a $20 \%$ energy window centred on $140 \mathrm{keV}$ on a General Electric DSTi gamma camera (Fairfield, CT, USA), equipped with low-energy and general purpose collimation. Recorded time varied from $5 \mathrm{~min}$ to $12 \mathrm{~min}$, depending on the patient morphology and cardiac rhythm. Processing was performed using the ECCAP software (GE Healthcare, Princeton, NJ, USA). Count-based left ventricle ejection fraction (LVEF) and RVEF were computed on semi-automatically drawn regions of interest, with two regions for the left ventricle (end-diastole and end-systole) and one region for the RV, drawn from the RV shape as seen at end diastole with the aid of Fourier amplitude and phase maps (fig. 1). The inter-observer reproducibility of RVEF measurements using ERNA has previously been evaluated as $<2 \%$ in our centre (data not shown). Normal RVEF values have been previously described at $>30 \%$ in our centre $[18,19]$. Physicians reading ERNA were blinded to patient characteristics at any time, and had access to baseline RVEF at the time of follow-up RVEF measurement.

\section{Follow-up evaluation}

The first visit after initiation of PAH therapy occurred 3-6 months after baseline in all patients as per guidelines. Patients lost to follow-up were censored at the time of their last visit. At data cut-off, the vital status was confirmed by review of the last medical record and was updated using a phone contact. End-points for the survival analysis were all-cause mortality and cardiovascular mortality.

\section{Data analysis}

Continuous variables are presented as mean $\pm \mathrm{SD}$, and categorical variables as percentages. Comparison of continuous variables between groups was performed using one-way ANOVA or non-parametric Mann-Whitney test for variables not normally distributed. Dichotomous variables were compared using the Chi-squared test. Correlations were assessed using linear regression analysis (Spearman or Pearson as appropriate). Estimates of survival were performed using the Kaplan-Meier estimator and the log-rank test, with the date of the RHC as baseline. Analyses were performed with two subgroups according to the median (RVEF $\geqslant 25 \%$ versus $<25 \%$ ) and the threshold of normal (RVEF $\geqslant 30 \%$ versus $<30 \%$ ). Patients who underwent lung transplantation were censored at the time of the operation. Sensitivity analyses were performed: 1) after the exclusion of patients who had a tricuspid annular plane systolic excursion (TAPSE) $<20 \mathrm{~mm}$ at baseline (reference for normal); 2) after exclusion of 51 patients who had a TAPSE $<16 \mathrm{~mm}$ at baseline (median of our cohort); and 3) only in patients with changes of RVEF superior to $2 \%$ during follow-up, in order to exclude those patients within the error boundaries of measurement (limit of inter-observer reproducibility of RVEF measurements). The relationship between survival and selected variables at baseline were further analysed with the univariate Cox regression method. The linearity and the proportional hazard assumption were tested and satisfied for all covariates. Variables with a p-value $<0.10$ at univariate analysis were included into multivariable Cox regression analysis (backward stepwise method) to examine the independent effect on survival of selected variables. To estimate the additional prognostic value of RVEF and TAPSE, we built a multivariable Cox model (adjustment 6MWD and sex) and introduced in turn RVEF or TAPSE (dichotomous or continuous variable). The predictive accuracy was determined by Harrell's C-index (proportion of patients in whom the predictions and outcomes are concordant). In addition, to test the information gain in predicting the outcome, different multivariate models were compared using the likelihood ratio test. Data analyses were conducted with SPSS 19.0.1 (SPSS, Chicago, IL, USA) and STATA 12 (Stata Corporation, College Station, TX, USA). A p-value $<0.05$ was considered statistically significant.

\section{Results}

\section{Study population}

Out of 222 consecutive incident patients with PAH (group 1) prospectively evaluated in our referral centre and included in the registry during the study period, 112 had idiopathic, familial or anorexigen-associated $\mathrm{PAH}$ and were eligible. Of them, 100 were included in the analysis (fig. 2). All patients were treatment 


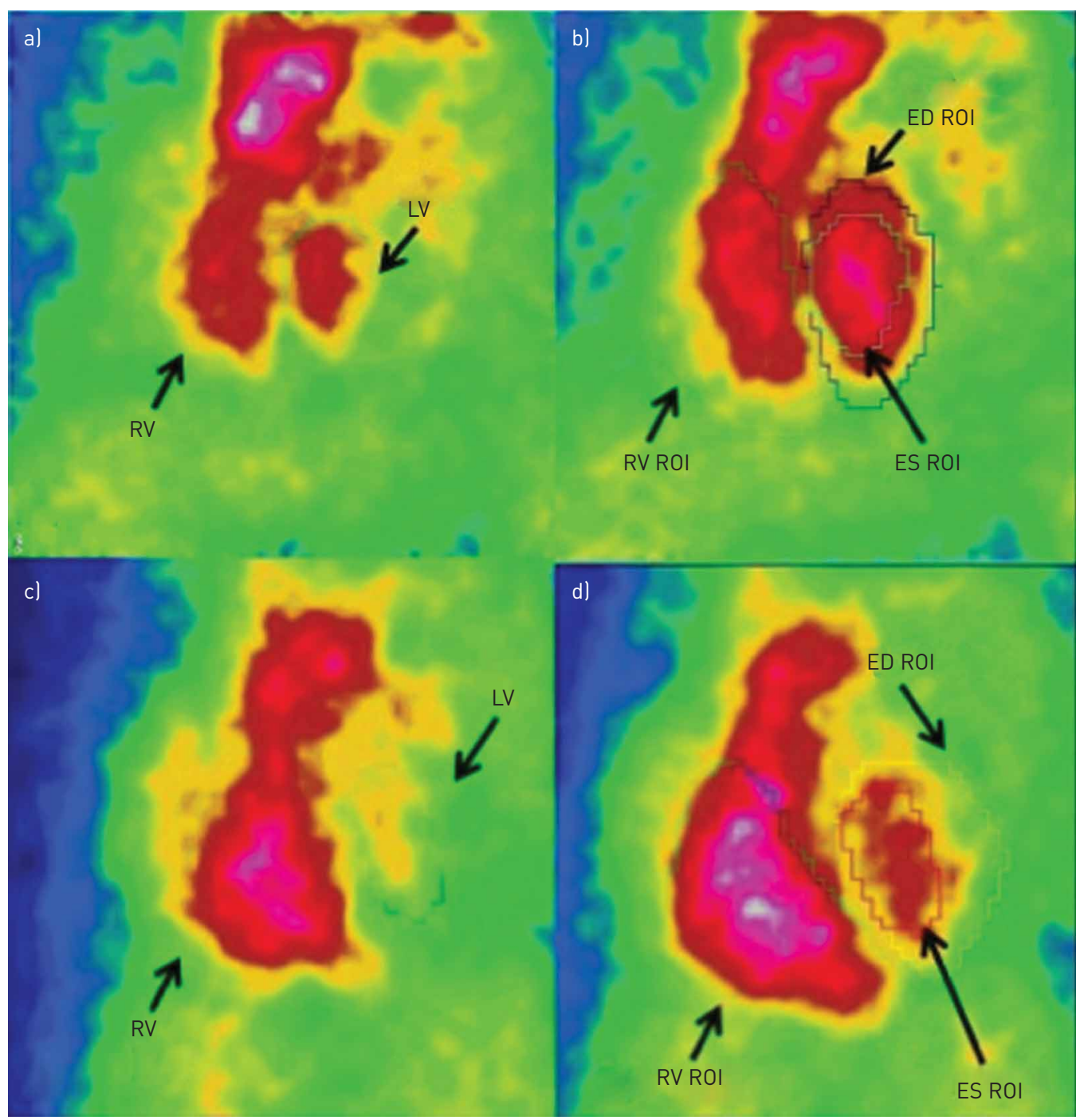

FIGURE 1 Typical a, c) end-systolic and b, d) end-diastolic blood pool pictures in two cases of pulmonary arterial hypertension. a, b) A patient with normal right ventricle ejection fraction (RVEF) $(>30 \%)$. c, d) A patient with an enlarged right ventricle (RV) and abnormal RVEF $(<25 \%)$. Count-based left ventricle ejection fraction and RVEF were computed on semi-automatically drawn regions of interest (ROI), end-diastole (ED) and end-systole (ES) for the left ventricle (LV) and ED for the RV.

naïve at baseline. In addition, 78 patients were evaluated after initiation of PAH therapy 3-6 months after baseline, including NYHA functional class, echocardiography, RHC and second assessment of RVEF using ERNA.

Patients had a mean \pm SD age of $57.2 \pm 18.6$ years, with a female:male ratio of $3: 2$ and a large predominance of idiopathic PAH. In addition, $56 \%$ of patients were in NYHA functional class III or IV at diagnosis (table 1). 6MWD, haemodynamics, echocardiography characteristics and PAH treatment at discharge are summarised in table 1 . At baseline, the mean \pm SD value of RVEF was $26.6 \pm 8.9 \%$ (median $25 \%$ ) while the LVEF was generally preserved (58.9 $\pm 9.4 \%)$. First-line therapy was initiated using oral medication in three-quarters of the cases.

\section{Characteristics of patients according to baseline RVEF}

As compared to those with baseline RVEF $\geqslant 25 \%$, patients with RVEF $<25 \%$ had similar demographic characteristics, aetiology, NYHA functional class, 6MWD and first-line therapy, but had significantly lower LVEF values, greater serum N-terminal pro-brain natriuretic peptide (NT-proBNP) levels, lower TAPSE at echocardiography, lower cardiac index and a trend for higher right atrial pressure (table 1). RVEF at baseline significantly correlated with TAPSE, tricuspid annulus peak systolic velocity (TAPSV), cardiac index and mixed venous oxygen saturation $\left(\mathrm{S}_{\mathrm{vO}}\right)$ and was inversely correlated with serum NT-proBNP 


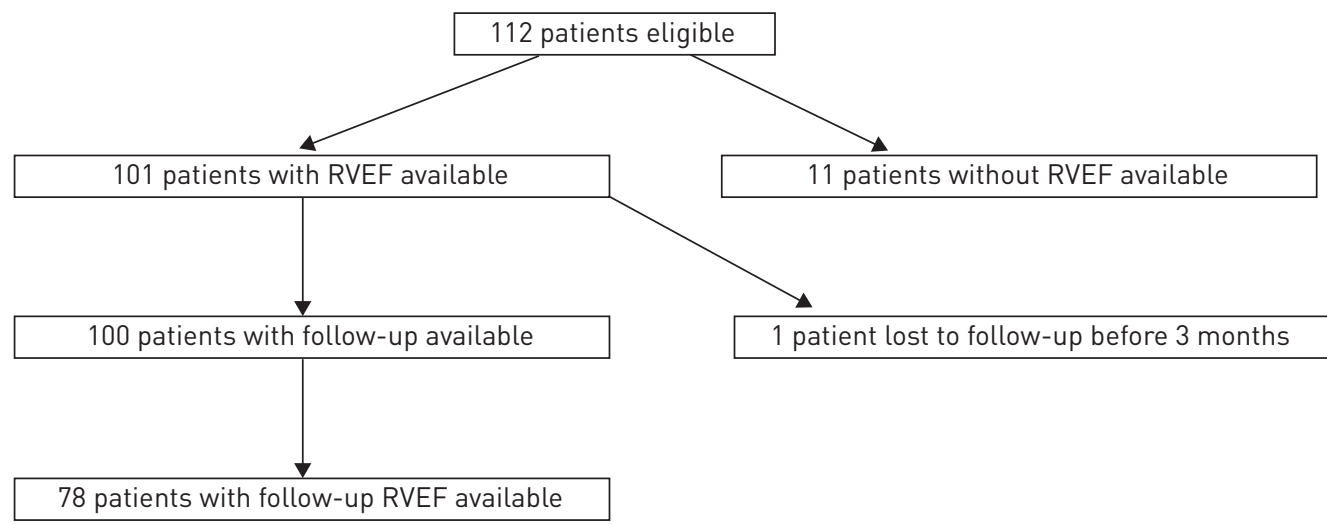

FIGURE 2 Flowchart summarising the generation of the cohort. RVEF: right ventricle ejection fraction.

\section{TABLE 1 Baseline characteristics of the study population}

\begin{tabular}{|c|c|c|c|c|}
\hline Characteristics & All & RVEF $<25 \%$ & RVEF $\geqslant 25 \%$ & p-value \\
\hline Subjects $\mathrm{n}$ & 100 & 50 & 50 & \\
\hline \multicolumn{5}{|l|}{ Demographics } \\
\hline Age years & $57.2 \pm 18.6$ & $58.0 \pm 18.8$ & $56.5 \pm 18.7$ & 0.683 \\
\hline Females/males $\mathrm{n}$ & $61 / 39$ & $26 / 24$ & $35 / 15$ & 0.065 \\
\hline Body mass index $\mathrm{kg} \cdot \mathrm{m}^{-2}$ & $26.9 \pm 6.4$ & $26.6 \pm 6.6$ & $27.2 \pm 6.2$ & 0.631 \\
\hline \multicolumn{5}{|l|}{ PAH group } \\
\hline Idiopathic & 79 (79) & $39(78)$ & $40(80)$ & 0.806 \\
\hline Familial & $4(4)$ & $2(4)$ & $2(4)$ & 0.967 \\
\hline Anorexigen-associated & $17(17)$ & $9(18)$ & $8(16)$ & 0.790 \\
\hline \multicolumn{5}{|l|}{ NYHA FC } \\
\hline I & $3(3)$ & $3(6)$ & $0(0)$ & 0.420 \\
\hline II & $41(41)$ & $17(34)$ & $24(48)$ & \\
\hline III & $51(51)$ & $26(52)$ & $25(50)$ & \\
\hline IV & $5(5)$ & $4(8)$ & $1(2)$ & \\
\hline 6-min walking distance $\mathrm{m}$ & $336 \pm 144$ & $335 \pm 116$ & $337 \pm 170$ & 0.962 \\
\hline \multicolumn{5}{|l|}{ ERNA \% } \\
\hline RVEF & $26.6 \pm 8.9$ & $18.1 \pm 4.3$ & $32.4 \pm 5.2$ & $<0.001$ \\
\hline LVEF & $58.9 \pm 9.4$ & $55.4 \pm 8.7$ & $62.3 \pm 8.8$ & $<0.001$ \\
\hline \multicolumn{5}{|l|}{ Echocardiography } \\
\hline TAPSE mm & $17.4 \pm 5.1$ & $16.2 \pm 5.0$ & $18.8 \pm 5.0$ & 0.018 \\
\hline TAPSV $\mathrm{cm} \cdot \mathrm{s}^{-1}$ & $10.6 \pm 2.6$ & $10.4 \pm 3.0$ & $11.0 \pm 2.1$ & 0.336 \\
\hline \multicolumn{5}{|l|}{ Biochemistry } \\
\hline NT-proBNP plasma levels $\mathrm{pg} \cdot \mathrm{mL}^{-1}$ & $2067 \pm 2110$ & $2582 \pm 2111$ & $1411 \pm 1964$ & 0.050 \\
\hline eGFR $\mathrm{mL} \cdot \mathrm{min}^{-1}$ & $76.8 \pm 28.8$ & $76.6 \pm 30.3$ & $76.9 \pm 27.5$ & 0.960 \\
\hline \multicolumn{5}{|l|}{ Haemodynamic data } \\
\hline Right atrial pressure $\mathrm{mmHg}$ & $7.8 \pm 5.2$ & $8.7 \pm 5.8$ & $6.7 \pm 4.3$ & 0.061 \\
\hline Mean PAP mmHg & $43.6 \pm 12.6$ & $44.8 \pm 12.2$ & $42.4 \pm 13.1$ & 0.349 \\
\hline PVR dyn $\cdot \mathrm{s} \cdot \mathrm{cm}^{-5}$ & $788 \pm 611$ & $886 \pm 714$ & $698 \pm 488$ & 0.141 \\
\hline Cardiac index $\mathrm{L} \cdot \mathrm{min}^{-1} \cdot \mathrm{m}^{-2}$ & $2.3 \pm 0.6$ & $2.2 \pm 0.6$ & $2.5 \pm 0.6$ & 0.020 \\
\hline \multicolumn{5}{|l|}{ Medication use } \\
\hline Endothelin receptor antagonist & $47(47)$ & $25(50)$ & $22(44)$ & 0.548 \\
\hline Phosphodiesterase- 5 inhibitor & $27(27)$ & $12(24)$ & $15(30)$ & 0.499 \\
\hline Intravenous epoprostenol & $9(9)$ & $7(14)$ & $2(4)$ & 0.081 \\
\hline Calcium antagonists & $2(2)$ & $2(4)$ & $0(0)$ & 0.153 \\
\hline
\end{tabular}

Data are presented as mean \pm SD or $n(\%)$, unless otherwise stated. RVEF: right ventricle ejection fraction; PAH: pulmonary arterial hypertension; NYHA: New York Heart Association; FC: functional class; ERNA: equilibrium radionuclide angiography; LVEF: left ventricle ejection fraction; TAPSE: tricuspid annular plane systolic excursion; TAPSV: tricuspid annulus peak systolic velocity; NT-proBNP: N-terminal pro-brain natriuretic peptide; eGFR: estimated glomerular filtration rate (modification in diet in renal disease formula); PAP: pulmonary arterial pressure; PVR: pulmonary vascular resistance. 
levels, right atrial pressure, mean PAP and PVR (table S1). Collectively, these results suggest that variables known to be associated with survival correlate with baseline RVEF in patients with PAH.

\section{Prognostic value of baseline variables}

After a median (interquartile range) follow-up of 4.1 (2.4-7.5) years, 41 patients had died (cardiovascular causes: $n=35$; sepsis: $n=2$; cancer: $n=2$; renal failure: $n=1$; severe bleeding: $n=1$ ). Kaplan-Meier survival estimates for the 100 patients were $86.0 \%$ at 1 year (95\% CI $81.5-89.5 \%$ ), $83.0 \%$ at 2 years (95\% CI $79.2-$ $86.8 \%$ ), $77.8 \%$ at 3 years (95\% CI $73.6-84.0 \%$ ), and $71.0 \%$ at 5 years (95\% CI $66.2-75.8 \%$ ). Two patients underwent lung transplantation.

In individual Cox regression analysis, lower age, absence of clinical evidence of RV failure, greater cardiac index, greater 6MWD, greater RVEF (dichotomous or continuous variable), TAPSE (continuous variable only), greater $\mathrm{S}_{\mathrm{vO}_{2}}$ and lower serum NT-proBNP values were significantly associated with a better overall survival (table 2). Multivariable analysis in a model adjusted for all continuous variables with a p-value $<0.10$ indicated that RVEF (continuous or dichotomous variable) was significantly associated with improved overall survival (HR 0.93, $\mathrm{p}=0.002$, and $\mathrm{HR} 0.26, \mathrm{p}=0.003$, respectively) (table 3 ). Univariable analysis further identified that the same variables were significantly associated with cardiovascular survival, and that the addition of female sex was also associated with greater cardiovascular survival (table 2). Multivariable analysis indicated that RVEF (continuous or dichotomous variable) was still significantly associated with improved cardiovascular survival (HR 0.93, $\mathrm{p}=0.007$ and HR 0.26, $\mathrm{p}=0.016$, respectively) (table 3). TAPSE (continuous or dichotomous variable) was not significant in the multivariable Cox regression model (cardiovascular or all-cause mortality). Using a censoring date at 5 years or 7 years of follow-up supports the same results (tables S2 and S3). Using the threshold of normal value (RVEF $\geqslant 30 \%$ versus $<30 \%$ ), the results were similar in univariate and in multivariate analysis (table S4).

Patients with RVEF $\geqslant 25 \%$ (median) at baseline had a better overall survival and cardiovascular survival than those with RVEF $<25 \%$ using Kaplan-Meier analysis ( $\mathrm{p}=0.010$ and $\mathrm{p}=0.029$, respectively) (fig. 3 ). Using a threshold of RVEF at $30 \%$ the results were quite similar ( $p=0.022$ for all-cause mortality and $\mathrm{p}=0.044$ for cardiovascular mortality, data not shown). The robustness of RVEF in predicting death was also tested in patients who had a normal value of TAPSE $(>20 \mathrm{~mm})$. In these subgroup analyses, the results were also statistically significant for all-cause mortality and only a trend was observed for

TABLE 2 Unadjusted hazard ratios and 95\% confidence intervals for right ventricle ejection fraction (RVEF) and major risk factors in predicting all-cause and cardiovascular mortality

\begin{tabular}{|c|c|c|c|c|}
\hline \multirow[t]{2}{*}{ Variables } & \multicolumn{2}{|c|}{ All-cause mortality } & \multicolumn{2}{|c|}{ Cardiovascular mortality } \\
\hline & $\operatorname{HR}(95 \% \mathrm{CI})$ & p-value & $\operatorname{HR}(95 \% \mathrm{CI})$ & p-value \\
\hline Age $>10$ years & $1.42(1.14-1.76)$ & 0.001 & $1.36(1.08-1.71)$ & 0.008 \\
\hline Male & $1.47(0.79-2.74)$ & 0.229 & $2.02(1.03-3.99)$ & 0.040 \\
\hline $\mathrm{BMI}>1 \mathrm{~kg} \cdot \mathrm{m}^{-2}$ & $0.99(0.93-1.04)$ & 0.648 & $1.00(0.94-1.06)$ & 0.958 \\
\hline NYHA FC III and IV & $1.51(0.80-2.88)$ & 0.206 & $1.46(0.73-2.92)$ & 0.287 \\
\hline Clinical evidence of RV failure & $2.43(1.27-4.66)$ & 0.008 & $2.39(1.18-4.84)$ & 0.016 \\
\hline $6 M W T>100 \mathrm{~m}$ & $0.60(0.47-0.76)$ & $<0.001$ & $0.60(0.46-0.78)$ & $<0.001$ \\
\hline Right atrial pressure $>1 \mathrm{mmHg}$ & $1.05(0.99-1.11)$ & 0.133 & $1.07(1.00-1.13)$ & 0.050 \\
\hline Mean PAP >1 mmHg & $0.99(0.97-1.02)$ & 0.513 & $1.00(0.98-1.03)$ & 0.822 \\
\hline Cardiac index $>1 \mathrm{~L} \cdot \mathrm{min}^{-1} \cdot \mathrm{m}^{-2}$ & $0.58(0.34-0.99)$ & 0.049 & $0.47(0.26-0.86)$ & 0.015 \\
\hline PVR $>1$ dyn $\cdot s \cdot \mathrm{cm}^{-5}$ & $1.00(1.00-1.00)$ & 0.487 & $1.00(1.00-1.00)$ & 0.268 \\
\hline TAPSE >1 mm & $0.92(0.85-1.00)$ & 0.044 & $0.90(0.83-0.098)$ & 0.018 \\
\hline TAPSE median & $0.94(0.47-1.92)$ & 0.874 & $1.26(0.57-2.82)$ & 0.569 \\
\hline TAPSE $\geqslant 20 \mathrm{~mm}$ versus $<20 \mathrm{~mm}$ & $1.13(0.43-2.920$ & 0.810 & $1.17(0.40-3.400$ & 0.770 \\
\hline TAPSV $>1 \mathrm{~cm} \cdot \mathrm{s}^{-1}$ & $0.91(0.77-1.07)$ & 0.264 & $0.88(0.74-1.06)$ & 0.183 \\
\hline Severe tricuspid regurgitation & $1.51(0.21-11.0)$ & 0.687 & $1.29(0.18-9.46)$ & 0.805 \\
\hline RVEF $>1 \%$ & $0.95(0.91-0.99$ & 0.006 & $0.95(0.91-0.99)$ & 0.011 \\
\hline RVEF $\geqslant 25 \%$ versus $<25 \%$ & $0.39(0.20-0.75)$ & 0.005 & $0.44(0.22-0.89)$ & 0.021 \\
\hline NT-proBNP $>1000 \mathrm{pg} \cdot \mathrm{mL}^{-1}$ & $1.23(1.02-1.47)$ & 0.027 & $1.28(1.06-1.54)$ & 0.010 \\
\hline $\mathrm{SvO}_{2}>1 \mathrm{mmHg}$ & $0.97(0.94-1.00)$ & 0.036 & $0.97(0.94-1.00)$ & 0.067 \\
\hline
\end{tabular}

BMI: body mass index; NYHA: New York Heart Association; FC: functional class; RV: right ventricle; 6MWT: 6-min walk test; PAP: pulmonary arterial pressure; PVR: pulmonary vascular resistance; TAPSE: tricuspid annular plane systolic excursion; TAPSV: tricuspid annulus peak systolic velocity. NT-proBNP: N-terminal pro-brain natriuretic peptide; $\mathrm{SvO}_{2}$ : mixed venous oxygen saturation. 
TABLE 3 Adjusted hazard ratios and 95\% confidence intervals for backward, stepwise Cox regression model, including all variables with a $p$-value $<0.10$ in a univariate analysis

\begin{tabular}{|c|c|c|c|c|}
\hline \multirow{2}{*}{ Variables } & \multicolumn{2}{|c|}{ All-cause mortality } & \multicolumn{2}{|c|}{ Cardiovascular mortality } \\
\hline & $\operatorname{HR}(95 \% \mathrm{CI})$ & p-value & $\operatorname{HR}(95 \% \mathrm{CI})$ & p-value \\
\hline \multicolumn{5}{|c|}{ Model with RVEF as continuous variable } \\
\hline RVEF > $1 \%$ & $0.93(0.89-0.98)$ & 0.002 & $0.93(0.88-0.98)$ & 0.007 \\
\hline Male & $2.14(0.95-4.85)$ & 0.068 & $3.46(1.40-8.51)$ & 0.007 \\
\hline $6 M W T>100 \mathrm{~m}$ & $0.45(0.33-0.62)$ & $<0.001$ & $0.41(0.29-0.60)$ & $<0.001$ \\
\hline \multicolumn{5}{|c|}{ Model with RVEF as dichotomous variable } \\
\hline RVEF $\geqslant 25 \%$ versus $<25 \%$ & $0.26(0.11-0.64)$ & 0.003 & $0.28(0.10-0.79)$ & 0.016 \\
\hline Male & $2.20(0.98-5.04)$ & 0.057 & $3.59(1.46-8.86)$ & 0.005 \\
\hline $6 \mathrm{MWT}>100 \mathrm{~m}$ & $0.44(0.31-0.62)$ & $<0.001$ & $0.41(0.28-0.79)$ & $<0.001$ \\
\hline
\end{tabular}

RVEF: right ventricle ejection fraction; 6MWT: 6-min walk test. RVEF was assessed with conventional planar equilibrium radionuclide angiography.

cardiovascular mortality with the same threshold of $25 \%$ (fig. S1) ( $p=0.027$ and $p=0.328$, respectively). We also observed a good predictive value of RVEF for patients who had a TAPSE superior to $16 \mathrm{~mm}$ (median) (all-cause mortality: $\mathrm{p}=0.010$; cardiovascular mortality: $\mathrm{p}=0.042$ ) (fig. S2).

The additional value of RVEF to TAPSE was tested using the C-index and the likelihood ratio test (table S5). The C-index increased slightly with RVEF in comparison to TAPSE. The information gain for RVEF in predicting all-cause and cardiovascular mortality was statistically significant in comparison with TAPSE $(\mathrm{p}<0.05$ with likelihood ratio test).

\section{Prognostic value of follow-up variables}

RVEF available at the first follow-up visit at 3-6 months from baseline and after initiation of PAH therapy in 78 patients (mean \pm SD $25.9 \pm 9.1 \%$ ) was not significantly different from baseline RVEF. The characteristics of patients with RVEF available at follow-up did not differ from those of patients where no RVEF was available (data not shown).

RVEF was stable or improved at follow-up in 56 (72\%) patients and decreased in the remaining 22 patients. As compared to patients who had lower RVEF at follow-up than at baseline, patients whose RVEF was stable or improved had similar demographic characteristics and aetiology of PAH; however, they had lower baseline RVEF and more severe haemodynamic characteristics at baseline (table S6). Furthermore, the change in RVEF from baseline to the first follow-up visit correlated significantly with the
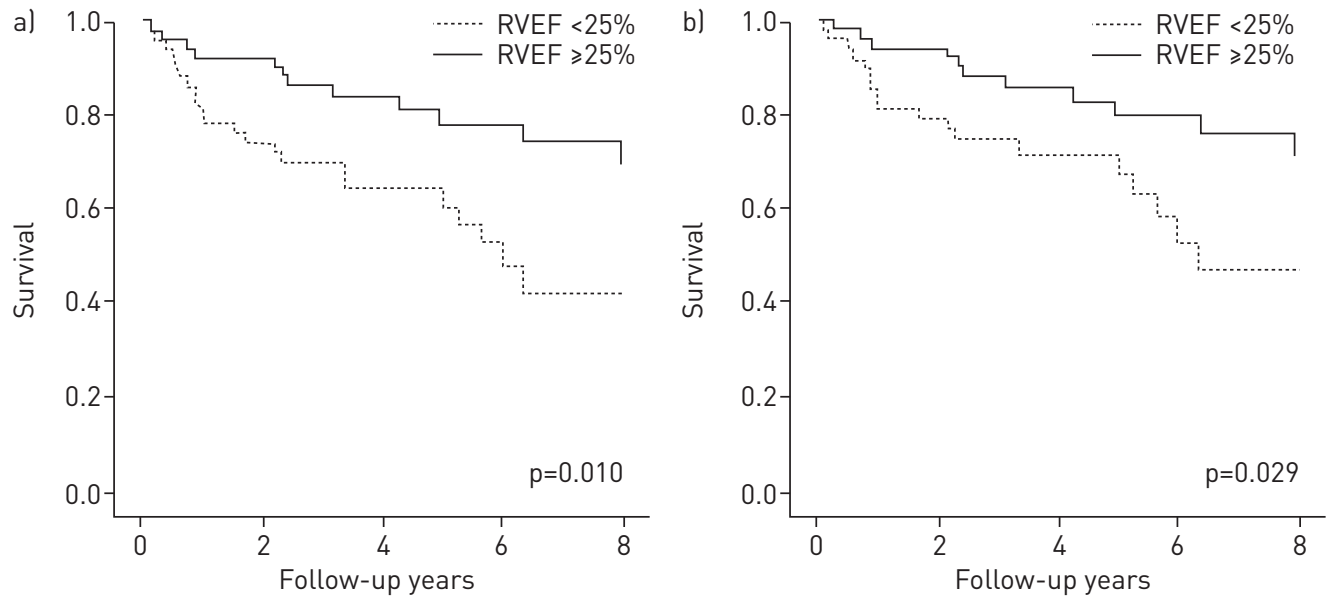

FIGURE 3 Survival estimates using the Kaplan-Meier method relative to the right ventricle ejection fraction (RVEF) at baseline admission for a) all-cause mortality and b) cardiovascular mortality. The 8 -year all-cause survival was $42.3 \%$ in patients with RVEF $<25 \%$ and $69.1 \%$ in patients with RVEF $\geqslant 25 \%$. The 5 -year cardiovascular survival was $47.1 \%$ in patients with RVEF $<25 \%$ and $70.5 \%$ in patients with RVEF $\geqslant 25 \%$. For RVEF $<25 \%$ the numbers at risk after $0,2,4,6$ and 8 years follow-up were 50,36, 20, 10, and 7, respectively. For RVEF $\geqslant 25 \%$ the numbers at risk after $0,2,4,6$ and 8 years follow-up were 50, 46, 33, 23 and 13, respectively. The p-values are from log-rank tests. 
change in cardiac index and $\mathrm{SvO}_{2}$, and negatively correlated with the change in right atrial pressure, mean PAP and PVR (table S7). Although the mean PVR decreased with medical therapy from baseline to the first follow-up visit, 79\% of patients with decreased PVR had stable or increased RVEF, while 53\% of patients with PVR that improved at follow-up also had decreased RVEF ( $p=0.017)$.

\section{Prognostic value of changes in RVEF with PAH therapy}

To test whether changes of various variables may predict the long-term prognosis of treated PAH patients, the variation in individual parameters between baseline and the first follow-up visit at 3-6 months (decrease versus stable or increase) were tested against survival using the Cox regression analysis. In univariable analysis, improvement in RVEF was the best predictor of subsequent overall survival $(\mathrm{p}=0.086)$ (table 4). Univariate analysis further identified that only an increase in RVEF was significantly associated with cardiovascular survival. Patients whose RVEF was stable or improved with PAH therapy, from baseline to the first follow-up visit, had a better all-cause and cardiovascular survival than those with a RVEF that decreased as compared to baseline by Kaplan-Meier analysis ( $p=0.076$ and $p=0.025$, respectively) (fig. 4). After exclusion of patients with RVEF changes inferior to $2 \%$, patients with an improvement of RVEF still had a better outcome (all-cause mortality: $n=54, p=0.039$; cardiovascular mortality: data not shown, $\mathrm{p}=0.009$ ).

\section{Discussion}

In this study, we demonstrated that the baseline RVEF assessed with conventional planar ERNA is an independent predictor of long-term overall survival in patients with idiopathic, familial, and anorexigen-associated PAH. Moreover, RVEF was still a strong predictor of outcome in patients with normal or quite normal TAPSE, assessed with transthoracic echocardiography. RVEF assessed with planar ERNA is able to identify high-risk patients, even when systolic function seems preserved using transthoracic echocardiography.

Our data further indicate that: 1) changes in RVEF at follow-up are associated with parallel changes in haemodynamic parameters, and are independently associated with long-term overall and cardiovascular survival; and 2) discrepant trends can be observed between PVR and RVEF at follow-up. These results were supported by comparable results for cardiovascular mortality in addition to correlations between RVEF and previously reported robust predictors of outcome in PAH, including cardiac index, right atrial pressure, TAPSE, NT-proBNP and $\mathrm{SvO}_{2}$ [4, 20-22]. Collectively, these observations suggest measurement of RVEF may be a useful tool to evaluate the long-term prognosis of patients with PAH. In addition, improvement in RVEF may be a more valuable, noninvasive indicator, than the standard echocardiography RV systolic parameters, as a means to predict survival in patients with recently initiated PAH therapy.

TABLE 4 Unadjusted hazard ratios and 95\% confidence intervals for major risk factors variation between baseline and the first follow-up visit at 3-6 months, in predicting all-cause and cardiovascular mortality

\begin{tabular}{|c|c|c|c|c|}
\hline \multirow[t]{2}{*}{ Variation" } & \multicolumn{2}{|c|}{ All-cause mortality } & \multicolumn{2}{|c|}{ Cardiovascular mortality } \\
\hline & $\operatorname{HR}(95 \% \mathrm{CI})$ & p-value & $\operatorname{HR}(95 \% \mathrm{CI})$ & p-value \\
\hline 6MWD & $1.36(0.44-4.22)$ & 0.595 & $1.63(0.50-5.36)$ & 0.417 \\
\hline Right atrial pressure & $0.43(0.12-1.57)$ & 0.199 & $0.36(0.08-1.73)$ & 0.202 \\
\hline Mean PAP & $2.71(0.83-8.80)$ & 0.097 & $1.83(0.52-6.49)$ & 0.349 \\
\hline Cardiac index & $1.04(0.34-3.18)$ & 0.947 & $2.60(0.55-12.27)$ & 0.277 \\
\hline PVR & $0.58(0.19-1.84)$ & 0.357 & $0.52(0.14-1.93)$ & 0.326 \\
\hline $\mathrm{SvO}_{2}$ & $2.63(0.76-9.11)$ & 0.126 & $3.62(0.81-16.18)$ & 0.093 \\
\hline TAPSE & $1.83(0.69-4.87)$ & 0.221 & $2.42(0.87-6.70)$ & 0.090 \\
\hline TAPSV & $1.41(0.57-3.51)$ & 0.461 & $1.18(0.43-3.25)$ & 0.753 \\
\hline RVEF & $2.43(0.88-6.71)$ & 0.086 & 3.25 (1.09-9.66) & 0.034 \\
\hline NT-proBNP & $0.76(0.17-3.41)$ & 0.721 & $0.56(0.11-2.79)$ & 0.481 \\
\hline eGFR & $1.21(0.52-2.79)$ & 0.660 & $1.17(0.47-2.87)$ & 0.737 \\
\hline
\end{tabular}

6MWD: 6-min walking distance; PAP: pulmonary artery pressure; PVR: pulmonary vascular resistance; $\mathrm{SvO}_{2}$ : mixed venous oxygen saturation; TAPSE: tricuspid annular plane systolic excursion; TAPSV: tricuspid annulus peak systolic velocity; RVEF: right ventricle ejection fraction; NT-proBNP: N-terminal pro-brain natriuretic peptide; eGFR: estimated glomerular filtration rate (modification in diet in renal disease formula). "\#: $n=78 ;{ }^{\text {n }}$ : decrease versus stable or increase. 

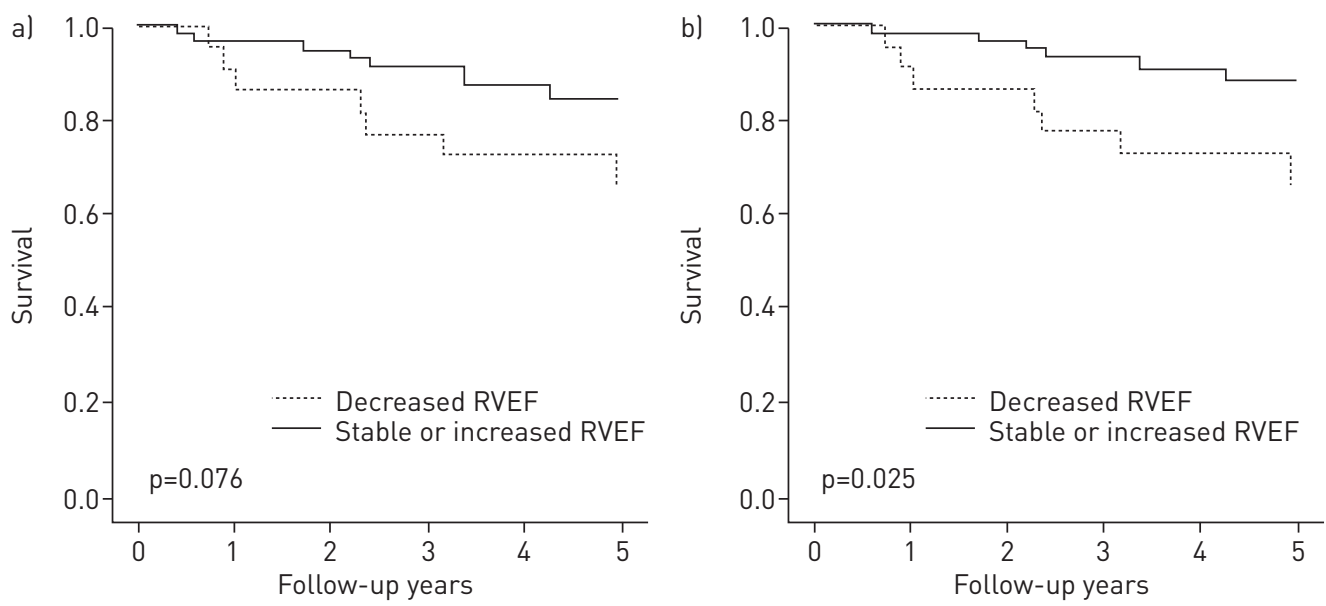

FIGURE 4 Survival estimates using the Kaplan-Meier method relative change in right ventricle ejection fraction (RVEF) from baseline to the first follow-up visit at 3-6 months following initiation of pulmonary arterial hypertension (PAH) therapy for a) all-cause survival and b) cardiovascular survival. The 5-year all-cause survival was $84.5 \%$ in patients with stable or increased RVEF and $65.9 \%$ in patients with decreased RVEF at first follow-up. The 5-year cardiovascular survival was $88.0 \%$ in patients with stable or increased RVEF and $65.9 \%$ in patients with decreased RVEF. For decreased RVEF the numbers at risk after $0,1,2,3,4$ and 5 years follow-up were 22, 20, 19, 17, 11 and 10, respectively. For stable or increased RVEF the numbers at risk after 0,1,2, 3, 4 and 5 years follow-up were 56, 54, 52, 46, 39 and 33, respectively. The p-values are from log-rank tests.

Other baseline variables relating to the RV function have previously been associated with overall survival in patients with PAH. Echocardiographic measurement of TAPSE at baseline predicted survival in a heterogeneous population of patients with precapillary $\mathrm{PH}$ (only one-third of whom had idiopathic $\mathrm{PAH}$ ) [21]. Similarly, RVEF assessed with cardiac MRI independently predicted long-term outcome in patients with PAH [22].

Little is known about which variables at follow-up are indicative of an appropriate response to therapy, and which situations should lead us to consider reinforcing therapy. Changes in NYHA functional class, cardiac index, $\mathrm{SvO}_{2}$ and NT-proBNP level better predict outcome than their baseline measurement [22, 23]. Improvement in RVEF at 3-6 months after therapy was the best predictor of cardiovascular and overall survival. VAN DE VEERDONK et al. [5] recently demonstrated, with the use of cardiac MRI, that RVEF can deteriorate with PAH therapy, with a decrease in RV function being associated with a poor outcome, irrespective of PVR variations. The present results, obtained with a different method, strongly indicate that the follow-up of RV function, after the initiation of therapy, may be a better predictor of outcome than the variation in PVR. Measurements of RVEF, therefore, need to be evaluated as a tool to adapt therapy in future dedicated studies. Although it is not known whether RV dysfunction is a marker of disease severity or reflects the failure of the RV to adapt to an increased afterload, it is increasingly considered that therapy targeting the RV function may be promising in PAH [24]. RVEF may also prove useful to identify subsets of patients who respond to PAH therapy. As an example, in a study of $\mathrm{PH}$ in the setting of idiopathic pulmonary fibrosis, treatment with sildenafil was associated with improvement in exercise capacity, however, only in the subgroup of patients with RV dysfunction at echocardiography [25]. RVEF might detect RV dysfunction earlier than RHC; whether repeated measurement of RVEF may ultimately contribute to fewer invasive investigations in the follow-up of patients treated for PAH requires further study.

Cardiac MRI is the current gold standard to assess RVEF [7]. Some of the limitations to the routine use of cardiac MRI include the requirement for breath holding and lying flat, claustrophobia, regular sinus rhythm, contraindication by some devices (infusion pumps, pacemaker and implantable cardioverter defibrillator), limited availability and expense. Both MRI and echocardiography require skilled physicians. We suggest that planar ERNA could be a valuable alternative to assess RV function in patients with PAH, with the advantage of very few contraindications (e.g. pregnancy and impossibility of intravenous injection), speed $(15 \mathrm{~min})$, reproducibility of measurement, being usable in the presence of cardiac arrhythmia, reasonable cost, and wide availability. A potential limitation to the use of conventional planar ERNA to assess RV function is related to irradiation, with the radiation dose of one conventional planar ERNA estimated at $7 \mathrm{mSv}$. As a comparison, the environmental yearly irradiation dose is $2.4 \mathrm{mSv}$ in France, and the radiation dose from a chest computed tomography can be up to $24 \mathrm{mSv}$. The cumulative estimated dose for five measurements is considered moderate; however with an increased risk of fatal cancer estimated at approximately $0.5 \%$ [26]. This risk should be considered, especially in younger patients. 
Although it was not compared to cardiac MRI, RVEF measured with conventional planar ERNA better predicted outcome than transthoracic echocardiographic regional indexes of RV function (TAPSE and TAPSV). These last parameters do not take into account the global systolic function of RV and especially the paradoxical motion of the ventricular septum, a limitation that could be overcome by more global echocardiographic indices of RV function. Hence, we have shown that measurements of RV function, using Doppler tissue imaging, independently predicts outcome in PAH [27]. Conventional planar ERNA is also particularly valuable in patients with a poor acoustic window limiting echocardiography, and further allows measurement of the LVEF, which may be affected by RV dilatation in patients with PAH [28].

The main technical limit to conventional planar ERNA is the relative difficulty to adequately separate, using the gamma camera signal, the RV from other cardiac structures [28]. However, RV enlargement in $\mathrm{PAH}$ enables an easier delineation of the region of interest. RVEF values assessed with conventional planar ERNA are known to be lower in absolute values than with other radionuclide techniques. However, reported correlations with gated first-pass RNA and ERNA are excellent, ranging from 0.85 to 0.95 [2931]. Given the high intrinsic reproducibility of ERNA measurements, it is unlikely that this may have affected the results presented here. Indeed, the reported intra- and inter-observer variability of ERNA measurements is very low $(2-3 \%)[30,31]$, comparable with our experience. The use of three-dimensional SPECT, rather than the ERNA method, may further refine measurement of RVEF in the near future.

A limitation of this study is that conventional planar ERNA, RHC and transthoracic echocardiography measurements, although prospectively acquired, were not obtained the same day, potentially resulting in measurements in different haemodynamic states. However, the time window between investigations was $<1$ week, which probably did not affect the results, and the data presented here reflects real-life conditions found in PAH management. While there was no difference in PAH therapy at baseline between patients with RVEF $<25 \%$ and those with RVEF $\geqslant 25 \%$, one could speculate that follow-up RVEF had probably guided modification of PAH therapy. As this method is not widely used in PAH, data were obtained at a single centre and the findings need to be validated at other centres, with comparison of RVEF to other parameters.

In conclusion, RVEF assessed with conventional planar ERNA at baseline is a strong predictor of long-term outcome in patients newly diagnosed with idiopathic, heritable and anorexigen-associated PAH. It compares favourably to transthoracic echocardiography to evaluate RV function and is a valuable alternative to cardiac MRI. Measurement of RVEF using ERNA after recent initiation of PAH-specific therapy further predicts outcome, and is a promising tool to guide management of patients with PAH.

\section{Acknowledgements}

We are grateful to Sabrina Zeghmar for data acquisition, Jean-Yves Bayle, Michèle Bertocchi, Lize Kiakouama, Fabrice Piegay, Julie Traclet and Catherine Paganon (Louis Pradel Hospital, Lyon, France) for patient care, and Gérald Simonneau (Bicêtre Hospital, Le Kremlin-Bicêtre, France) for help with the French pulmonary hypertension registry.

\section{References}

1 Humbert M, Sitbon O, Simonneau G. Treatment of pulmonary arterial hypertension. N Engl J Med 2004; 351: $1425-1436$.

2 Humbert M, Sitbon O, Chaouat A, et al. Survival in patients with idiopathic, familial, and anorexigen-associated pulmonary arterial hypertension in the modern management era. Circulation 2010; 122: 156-163.

3 Vonk Noordegraaf A, Galiè N. The role of the right ventricle in pulmonary arterial hypertension. Eur Respir Rev 2011; 20: 243-253.

4 D'Alonzo GE, Barst RJ, Ayres SM, et al. Survival in patients with primary pulmonary hypertension: results from a national prospective registry. Ann Intern Med 1991; 115: 343-349.

5 van de Veerdonk MC, Kind T, Marcus JT, et al. Progressive right ventricular dysfunction in patients with pulmonary arterial hypertension responding to therapy. J Am Coll Cardiol 2011; 58: 2511-2519.

6 Vonk-Noordegraaf A, Westerhof N. Describing right ventricular function. Eur Respir J 2013; 41: 1419-1423.

7 Galiè N, Hoeper MM, Humbert M, et al. Guidelines for the diagnosis and treatment of pulmonary hypertension. Eur Respir J 2009; 34: 1219-1263.

8 Torbicki A. Cardiac magnetic resonance in pulmonary arterial hypertension: a step in the right direction. Eur Heart J 2007; 28: 1187-1189.

9 van Wolferen SA, Marcus JT, Boonstra A, et al. Prognostic value of right ventricular mass, volume, and function in idiopathic pulmonary arterial hypertension. Eur Heart J 2007; 28: 1250-1257.

10 Nichols K, Saouaf R, Ababneh AA, et al. Validation of SPECT equilibrium radionuclide angiographic right ventricular parameters by cardiac magnetic resonance imaging. J Nucl Cardiol 2002; 9: 153-160.

11 Humbert M, Sitbon O, Chaouat A, et al. Pulmonary arterial hypertension in France: results from a national registry. Am J Respir Crit Care Med 2006; 173: 1023-1030.

12 Miller DP, Gomberg-Maitland M, Humbert M. Survivor bias and risk assessment. Eur Respir J 2012; 40: 530-532.

13 Simonneau G, Robbins IM, Beghetti M, et al. Updated clinical classification of pulmonary hypertension. J Am Coll Cardiol 2009; 54: Suppl., S43-S54.

14 Sitbon O, Humbert M, Simonneau G. Les Traitements de l' hypertension artérielle pulmonaire a l' heure de la T2A. Recommandations du Groupe de Travail Maladies Vasculaires Pulmonaires de la Société de Pneumologie de 
langue Française [Treatment for pulmonary arterial hypertension under the new French hospital financing system. Recommendations of the Pulmonary Vascular Diseases Working Group of the French Society of Pulmonary Medicine]. Presse Med 2005; 34:1456-1464.

15 Sitbon $\mathrm{O}$, Humbert M, Jais X, et al. Long-term response to calcium channel blockers in idiopathic pulmonary arterial hypertension. Circulation 2005; 111: 3105-3111.

16 ATS statement: Guidelines for the six-minute walk test. Am J Respir Crit Care Med 2002; 166: 111-117.

17 Groch MW, DePuey EG, Belzberg AC, et al. Planar imaging versus gated blood-pool SPECT for the assessment of ventricular performance: a multicenter study. J Nucl Med 2001; 42: 1773-1779.

18 Merabet Y, Bontemps L, Chevalier P, et al. Scintigraphic spectrum of a patient population with suspected arrhythmogenic right ventricular dysplasia. Int J Cardiovasc Imaging 2012; 28: 1267-1277.

19 Bontemps L, Merabet Y, Chevalier P, et al. Clinical validation of the planar radionuclide ventriculography in patients with right ventricular dysfunction. Rev Esp Med Nucl Imagen Mol 2013; 32: 364-370.

20 Eysmann SB, Palevsky HI, Reichek N, et al. Two-dimensional Doppler-echocardiographic and cardiac catheterization correlates of survival in primary pulmonary hypertension. Circulation 1989; 80: 353-360.

21 Forfia PR, Fisher MR, Mathai SC, et al. Tricuspid annular displacement predicts survival in pulmonary hypertension. Am J Respir Crit Care Med 2006; 174: 1034-1041.

22 Nickel N, Golpon H, Greer M, et al. The prognostic impact of follow-up assessments in patients with idiopathic pulmonary arterial hypertension. Eur Respir J 2012; 39: 589-596.

23 Kawut SM, Horn EM, Berekashvili KK, et al. New predictors of outcome in idiopathic pulmonary arterial hypertension. Am J Cardiol 2005; 95: 199-203.

24 Hemnes AR, Kawut SM. The right ventricle in pulmonary hypertension: from dogma to data. Am J Respir Crit Care Med 2010; 182: 586-588.

25 Han MK, Bach DS, Hagan PG, et al. Sildenafil preserves exercise capacity in patients with idiopathic pulmonary fibrosis and right-sided ventricular dysfunction. Chest 2013; 143: 1699-1708.

26 Verdun FR, Bochud F, Gundinchet F, et al. Quality initiatives radiation risk: what you should know to tell your patient. Radiographics 2008; 28: 1807-1816.

27 Ernande L, Cottin V, Leroux PY Right isovolumic contraction velocity predicts survival in pulmonary hypertension. J Am Soc Echocardiogr 2013; 26: 297-306.

28 Gan C, Lankhaar JW, Marcus JT, et al. Impaired left ventricular filling due to right-to-left ventricular interaction in patients with pulmonary arterial hypertension. Am J Physiol Heart Circ Physiol 2006; 290: H1528-H1533.

29 Schulman DS. Assessment of the right ventricle with radionuclide techniques. J Nucl Cardiol 1996; 3: 253-264.

30 Maddahi J, Berman DS, Matsuoka DT, et al. A new technique for assessing right ventricular ejection fraction using rapid multiple-gated equilibrium cardiac blood pool scintigraphy: description, validation and findings in chronic coronary artery disease. Circulation 1979; 60: 581-589.

31 Morrison D, Marshall J, Wright AI, et al. An improved method of right ventricular gated equilibrium blood pool radionuclide ventriculography. Chest 1982; 82: 607-614. 